\title{
Sequencing of the type species of Arthopyrenia places Arthopyreniaceae as a synonym of Trypetheliaceae
}

\author{
Thiyagaraja $V^{1,2,3,4,5}$, Lücking $\mathrm{R}^{6}$, Ertz $\mathrm{D}^{7,8}$, Coppins $\mathrm{BJ}^{9}$, Wanasinghe $\mathrm{DN}^{4,5}$, \\ Karunarathna $\mathrm{SC}^{4,5}$, Suwannarach $\mathrm{N}^{10}$, To-Anun $\mathrm{C}^{1}$, Cheewangkoon $\mathrm{R}^{1,11^{*}}$ and \\ Hyde $\mathrm{KD}^{1,2,3,4^{*}}$
}

\author{
${ }^{1}$ Department of Entomology and Plant Pathology, Faculty of Agriculture, Chiang Mai University, Chiang Mai 50200, \\ Thailand \\ ${ }^{2}$ Innovative Institute of Plant Health, Zhongkai University of Agriculture and Engineering, Haizhu District, \\ Guangzhou, Guangdong 510225, People's Republic of China \\ ${ }^{3}$ Centre of Excellence in Fungal Research, Mae Fah Luang University, Chiang Rai 57100, Thailand \\ ${ }^{4}$ Centre for Mountain Futures (CMF), CAS Key Laboratory for Plant Biodiversity and Biogeography of East Asia \\ (KLPB), Kunming Institute of Botany, Chinese Academy of Science, Kunming 650201, Yunnan, People's Republic of \\ China \\ ${ }^{5}$ CIFOR-ICRAF China Program, World Agroforestry (ICRAF), Kunming 650201, People's Republic of China \\ ${ }^{6}$ Botanischer Garten und Botanisches Museum, Freie Universität Berlin, Königin-Luise-Str. 6-8, 14195 Berlin, \\ Germany \\ ${ }^{7}$ Research Department, Meise Botanic Garden, Nieuwelaan 38, BE-1860 Meise, Belgium \\ ${ }^{8}$ Fédération Wallonie-Bruxelles, Service Général de l'Enseignement Supérieur et de la Recherche Scientifique, Rue A. \\ Lavallée 1, BE-1080 Bruxelles, Belgium \\ ${ }^{9}$ Royal Botanic Garden Edinburgh, Edinburgh EH3 5LR, UK \\ ${ }^{10}$ Research Center of Microbial Diversity and Sustainable Utilization, Chiang Mai University, Chiang Mai 50200, \\ Thailand \\ ${ }^{11}$ Innovative Agriculture Research Center, Chiang Mai University, Chiang Mai 50200, Thailand
}

Thiyagaraja V, Lücking R, Ertz D, Coppins BJ, Wanasinghe DN, Karunarathna SC, Suwannarach N, To-Anun C, Cheewangkoon R, Hyde KD 2021 - Sequencing of the type species of Arthopyrenia places Arthopyreniaceae as a synonym of Trypetheliaceae. Mycosphere 12(1), 993-1011, Doi 10.5943/mycosphere/12/1/10

\begin{abstract}
Arthopyrenia sensu lato comprises lichenicolous, lichenized and non-lichenized saprotrophic species; however, the lifestyle of several taxa as either lichenized or saprotrophic remains unclear. The systematic position of the genus was so far unresolved: while sequenced species appeared in different clades within Dothideomycetes, the type species, A. cerasi, had no molecular data so far. In lieu of sequence data, the family Arthopyreniaceae was assigned to Pleosporales, whereas tropical, lichenized species were reclassified in Constrictolumina and Macroconstrictolumina, shown to belong in Trypetheliaceae (Trypetheliales). In this study, the generic type, A. cerasi, has been sequenced for the first time. Maximum likelihood and Bayesian phylogenetic analyses using mtSSU and nuLSU sequences recovered Arthopyrenia sensu stricto as an early diverging lineage within Trypetheliaceae, separate from Constrictolumina and Macroconstrictolumina but in the same clade as the temperate, non-lichenized Julella fallaciosa. Therefore, Arthopyreniaceae is here synonymized under Trypetheliaceae and the taxonomic placement of its type species is discussed based on morphological and phylogenetic evidence. Our phylogenetic results further support the
\end{abstract}


polyphyly of non-lichenized, temperate species of Arthopyrenia and Julella sensu lato. Consequently, Julella fallaciosa is transferred to Arthopyrenia and its close relationship with A. cerasi is discussed. We also conducted ancestor character state analysis to reconstruct lifestyle changes within Trypetheliales using Bayes Traits and Bayesian Binary MCMC approaches.

Keywords - ancestral character state analyses - Julella - lifestyles - non-lichens - phylogeny taxonomy

\section{Introduction}

Dothideomycetes is the largest class in Ascomycota (Hyde et al. 2013, Hongsanan et al. 2020a, b). It includes mostly saprotrophic or parasitic fungi but also lichenized lineages that evolved independently relative to other large lichenized classes, such as Arthoniomycetes and Lecanoromycetes (Lutzoni et al. 2001, Gueidan et al. 2008, Nelsen et al. 2009, 2011, Schoch et al. 2009). Several lichenized lineages in Dothideomycetes also include non-lichenized saprotrophic taxa, which either appear as early diverging lineages or have been derived through loss of lichenization. These potentially secondarily saprotrophic fungi are primarily associated with Arthopyreniaceae, Lichenotheliaceae, Monoblastiaceae, Mycoporaceae, Trypetheliaceae and Xanthopyreniaceae (Coppins \& Aptroot 2008, Hyde et al. 2013, Aptroot et al. 2016, Pérez-Ortega et al. 2016, Hongsanan et al. 2020b).

Arthopyreniaceae was established by Watson (1929) to encompass lichenized fungi with trentepohlioid photobiont and branched and anastomosing paraphyses. Watson (1929) originally included 13 genera within the family; however, over time only Arthopyrenia remained in this family, while other genera were transferred to different families, orders and classes or were subsumed under synonymy of genera in other lineages (Hyde et al. 2013, 2016, Lücking et al. 2017). In the most recent classification, Arthopyreniaceae included only two genera, viz. Arthopyrenia and Mycomicrothelia, and was assigned to Pleosporales (Hyde et al. 2013, Wijayawardene et al. 2017, 2018, 2020, Hongsanan et al. 2020a). This classification was, however, tentative, since the type species of both genera have not yet been sequenced (Liu et al. 2014, Hyde et al. 2016). Phylogenetic studies placed many species previously classified in Arthopyrenia or Mycomicrothelia within Trypetheliales (Nelsen et al. 2009, 2011, 2014, Aptroot \& Lücking 2016, Hyde et al. 2016, Hongsanan et al. 2020b). Arthopyrenia needs critical study with regard to its placement and delimitation, for which the phylogenetic placement of the type species is essential (Hyde et al. 2013, Nelsen et al. 2011, 2014).

Species of Arthopyreniaceae occur in terrestrial habitats and are distributed mainly in tropical and temperate regions, known from both sexual and asexual states (Coppins 1988, Hyde et al. 2013). Arthopyrenia itself was introduced by Massalongo (1852) for an assemblage of lichenized, lichenicolous and non-lichenized fungi (Zahlbruckner 1921). It was restudied in detail by Harris (1973, 1975, 1995), Tucker \& Harris (1980) and Coppins (1988). Index Fungorum (2021) shows nearly 880 species epithets under this genus name. However, many species have been transferred to Monoblastiaceae, Naetrocymbaceae, Porinaceae, Strigulaceae and Trypetheliaceae and the taxonomy of some species remains uncertain (Coppins 1988). In a more limited sense, Arthopyrenia presently comprises 53 species (Species Fungorum 2021), which are chiefly known from temperate regions (Harris \& Tripp 2013, Hyde et al. 2013). The taxa are characterized mainly by hamathecium characteristics, such as densely arranged paraphysoids developing from both ends and a $\mathrm{K}+$ sordid-green perithecial wall (Hyde et al. 2013, Hongsanan et al. 2020a). Many Mycomicrothelia species have been transferred to Bogoriella and Pseudobogoriella (Aptroot \& Lücking 2016, Hongsanan et al. 2020b) and only nine species are currently retained in Mycomicrothelia (Species Fungorum 2021).

In this study, we aim to establish the phylogenetic position of Arthopyrenia sensu stricto by sequencing of the type species, A. cerasi and thereby reassess its relationship with the already sequenced Arthopyrenia species and with sequenced species of the genus Julella. We also provide detailed morphological descriptions and molecular data for Alloarthopyrenia italica and 
Pseudopyrenula endoxanthoides, two confirmed members of Trypetheliaceae, based on new material. Ancestor character state analysis was performed to reconstruct lifestyle changes in Trypetheliales using Bayes Traits and Bayesian Binary MCMC approaches.

\section{Material \& Methods}

\section{Phenotypic analyses}

Fresh material of Arthopyrenia cerasi and Arthopyrenia italica was collected in Europe, whereas Pseudopyrenula endoxanthoides was collected in Thailand. Specimens were examined using a Motic SMZ 168 dissecting microscope. Hand sections of the ascomata were mounted in water, 5\% $\mathrm{KOH}$ and Lugol's solution to examine micro-morphological characteristics. Macromorphological structures were observed with a stereo microscope (Motic SMZ-168) and photographed with Zeiss discovery v8 stereomicroscope (Carl Zeiss, Jena, Germany). Micromorphological details were studied using a Nikon ECLIPSE 80i compound microscope fitted with a Canon 550D digital camera. For Arthopyrenia cerasi, macroscopic photographs were made with a Keyence VHX-5000 Digital Microscope and a VH-Z20R/W/T lens, while microscopic photographs were prepared using an Olympus BX51 compound microscope, fitted with an Olympus SC50 digital camera.

All microscopic measurements were done in water-mounted slides and made with Tarosoft Image Frame Work (09.0.7). Images used for figures were processed with Adobe Photoshop CS6 Extended 10.0 software (Adobe Systems, USA). The material of Arthopyrenia cerasi is deposited in the Royal Botanic Garden Edinburgh (E) and in the Meise Botanic Garden (BR). The material of Arthopyrenia italica and Pseudopyrenula endoxanthoides is deposited in the herbarium of Mae Fah Luang University (MFLU), Chiang Rai, Thailand. Index Fungorum and Faces of Fungi numbers were registered following Index Fungorum (2021) and Jayasiri et al. (2015).

\section{DNA extraction, PCR amplification and sequencing}

DNA isolation of Arthopyrenia cerasi was carried out from hand-made sections of ascomata by the direct PCR method as described in Ertz et al. (2015, 2018). For the other taxa, an E.Z.N.A. ( Forensic DAT (D3591 - 01, Omega Bio - Tek) DNA extraction kit was used to extract DNA from fruiting structures by following the manufacturer's instructions. DNA samples that were intended for use as a template for PCR were stored at $4^{\circ} \mathrm{C}$ for use in regular work and duplicated at $-20^{\circ} \mathrm{C}$ for long-term storage. DNA sequence data were obtained from partial sequences of ribosomal and mitochondrial coding genes to generate following gene markers: Mitochondrial small subunit spacers (12S, mtSSU) and large subunit nuclear rDNA (28S, LSU) and amplified with primer pairs mrSSU1 and mrSSU3R (Zoller et al. 1999) and LR0R and LR5 (Vilgalys \& Hester 1990) respectively. The PCR amplification was performed following Ertz et al. (2018) for A. cerasi and Thiyagaraja et al. (2021) for other taxa using a final volume of $25 \mu 1$, comprised of 2.0 $\mu 1$ of DNA template, $1 \mu 1$ of each forward and reverse primers, $12.5 \mu 1$ of Taq PCR Super Mix (mixture of Easy Taq TM DNA Polymerase, dNTPs, obtained buffer (Beijing Trans Gen Biotech Co., Chaoyang District, Beijing, PR China)) and $8.5 \mu$ l of sterilized water. PCR products were examined on $1 \%$ agarose electrophoresis gels and stained with ethidium bromide and sent for sequencing to Macrogen ${ }^{\circledR}$ for Arthopyrenia cerasi and to Tsingke (Yunnan Province, P.R. China) for the other taxa. New nucleotide sequence data acquired were deposited in GenBank (Table 1). Alignments and phylogenetic trees were submitted to TreeBASE under submission number 28222.

\section{Taxon sampling}

The BLAST search engine of the National Centre for Biotechnology Information (NCBI) was used for the preliminary identification of newly generated DNA sequences (https://www.ncbi.nlm.nih.gov). After confirming that all newly generated sequences represented Trypetheliaceae, selected sequences of Trypetheliales (including Polycoccaceae) were retrieved from GenBank. Initially, phylogenetic analyses were conducted for Trypetheliales and their close 
relatives following Hongsanan et al. (2020b). Outgroup taxa were selected from representatives of Dothideales, Capnodiales and Myriangiales (Hongsanan et al. 2020b). The final combined alignment comprised 118 terminals (Table 1), 83 of which had mtSSU and 109 of which had nuLSU sequence data.

\section{Phylogenetic analyses and species recognition}

Phylogenetic analyses of both single marker and concatenated data were performed under maximum likelihood and Bayesian inference. Sequences of the mtSSU and the nuLSU were aligned separately using MAFFT v. 7 (http://mafft.cbrc.jp/alignment/ server/index.html, Katoh et al. 2019). Terminal ends of sequences and ambiguous regions were trimmed manually using BioEdit v. 7.0.5.2 (Hall 1999). The phylogenetic web tool "ALTER" (Glez-Peña et al. 2010) was used to convert sequence alignment from FASTA to PHYLIP for RAxML analysis and from FASTA to NEXUS format for Bayesian analysis. Best models for the BI approach was established independently for each locus using MrModeltest v.2.2 (Nylander 2004). The ML tree was generated using the RAxML-HPC2 on XSEDE (8.2.8) (Stamatakis 2014) on the CIPRES Science Gateway platform (Miller et al. 2010), with 1000 separate runs. MrBayes v. 3.1.2 was used to perform Bayesian analysis (Huelsenbeck \& Ronquist 2001). Markov Chain Monte Carlo sampling (MCMC), was run for 50000000 generations and trees were sampled every $100^{\text {th }}$ generations. The first $10 \%$ of trees that represented the burn-in phase were discarded, and only the remaining $90 \%$ of trees were used for calculating posterior probabilities (PP) for the majority rule consensus tree. No conflict was detected between individual markers and so the final analyses were performed on the concatenated data set. Resulting trees were drawn in FigTree v1.4.0 (Rambaut 2014), then copied to Microsoft PowerPoint 2013 and converted to jpeg files using Adobe Photoshop CS6 Extended 10.0 (Adobe Systems. U.S.A.).

\section{Ancestral character state analyses}

Ancestral character state analyses were carried out to reconstruct the evolutionary relationship of lifestyle changes in Trypetheliales. The following states were established: lichenized, non-lichenized saprotrophic, borderline lichenized (i.e. weakly lichenized, with a whitish, thallus like area but few photobiont cells only), lichenicolous, and plant-pathogenic. The platform Reconstruct Ancestral State in Phylogenies (RASP 3.2.1) was used to construct ancestral character analyses, using the two approaches of Bayes Traits and Bayesian Binary MCMC based on the ML tree (Yu et al. 2015, 2019). Both approaches were performed and visualized in RASP 3.2.1 using settings as follows: 1010000 iterations for Bayes Traits with a burn-in of 10 000, sampling 1000 trees and with 10 ML trees; 50000 generations for Bayesian Binary MCMC, with 10 chains, a sample frequency of 100 , a temperature of 0.1 , state frequencies fixed (JC), and among-site rate variation equal. The trees were edited using Microsoft PowerPoint 2013 and converted to jpeg files using Adobe Photoshop CS6 Extended 10.0 (Adobe Systems. U.S.A.).

Table 1 Taxa names, strain numbers and GenBank accession numbers of the taxa used for phylogenetic analyses. The newly generated sequences are indicated in black boldface

\begin{tabular}{llcc}
\hline \multirow{2}{*}{ Taxa } & \multirow{2}{*}{ Strain } & \multicolumn{2}{c}{ GenBank Accessions } \\
\cline { 3 - 4 } & & mtSSU & nuLSU \\
\hline Alloarthopyrenia italica & MFLU 15-0399 & KX655555 & KX655550 \\
Alloarthopyrenia italica & MFLU 17-1689 & MZ221609 & - \\
Aptrootia elatior & MPN560B & KM453821 & KM453754 \\
Aptrootia robusta & MPN235B & KM453822 & KM453755 \\
Aptrootia terricola & F 17211 & DQ328995 & - \\
Architrypethelium lauropaluanum & MPN48 & KX215566 & KX215605 \\
Architrypethelium nitens & MPN257 & KM453823 & KM453757 \\
Architrypethelium uberinum & MPN489 & - & KM453758 \\
Arthopyrenia cerasi & Coppins 25807 (BR) & MZ221617 & - \\
\hline
\end{tabular}


Table 1 Continued.

\begin{tabular}{|c|c|c|c|}
\hline \multirow{2}{*}{ Taxa } & \multirow{2}{*}{ Strain } & \multicolumn{2}{|c|}{ GenBank Accessions } \\
\hline & & mtSSU & nuLSU \\
\hline Arthopyrenia fallaciosa & MPN141 & JN887411 & JN887399 \\
\hline Arthopyrenia fallaciosa & MPN547 & JN887412 & JN887400 \\
\hline Arthopyrenia salicis & CBS 368.94 & AY538345 & AY538339 \\
\hline Astrothelium aeneum & MPN302 & - & KX215606 \\
\hline Astrothelium aff. crassum & MPN335 & KM453827 & KM453761 \\
\hline Astrothelium confusum & Nelsen 4004a (F) & GU327685 & GU327710 \\
\hline Astrothelium croceum & Nelsen 211D (F) & KX215567 & KX215611 \\
\hline Astrothelium leuсосопicum & MPN42 & KM453830 & KM453764 \\
\hline Astrothelium macrocarpum & MPN260 & KM453829 & KM453763 \\
\hline Astrothelium macrocarpum & NSR6 & AB759879 & LC127402 \\
\hline Astrothelium neglectum & TAK8 & LC128025 & LC127410 \\
\hline Astrothelium neovariolosum & KY777 & LC128023 & LC127408 \\
\hline Astrothelium nitidiusculum & MPN704 & KM453868 & KM453804 \\
\hline Astrothelium norisianum & MPN52C & KM453848 & KM453783 \\
\hline Astrothelium perspersum & AFTOL2099 & GU561848 & FJ267701 \\
\hline Astrothelium siamense & KRB139 & LC128021 & LC127406 \\
\hline Astrothelium subcatervarium & Nelsen 4009a (F) & GU327707 & GU327729 \\
\hline Astrothelium variolosum & MPN41 & KX215585 & KX215662 \\
\hline Bathelium lineare & MPN741 & KM453839 & KM453774 \\
\hline Bathelium porinosporum & MPN744 & KX215586 & KX215665 \\
\hline Bathelium porinosporum & MPN747 & KX215587 & KX215667 \\
\hline Bathelium tuberculosum & MPN112 & _- & KX215668 \\
\hline Bathelium tuberculosum & MPN113 & - & KX215669 \\
\hline Bathelium tuberculosum & MPN81 & KM453842 & KM453777 \\
\hline Bogoriella oleosa & MPN700 & KM453857 & KM453794 \\
\hline Bogoriella oleosa & Nelsen 4007a (F) & GU327697 & GU327721 \\
\hline Capnodium coffeicola & MFLUCC 15-0206 & - & KU358920 \\
\hline Chaetothyriothecium elegans & СРC 21375 & - & NG 058861 \\
\hline Constrictolumina cinchonae & Lücking 29583 & JN872349 & JN872351 \\
\hline Constrictolumina cinchonae & MPN417 & KM453825 & KM453759 \\
\hline Constrictolumina planorbis & MPN330 & - & KX215670 \\
\hline Constrictolumina planorbis & MPN331 & - & KX215671 \\
\hline Constrictolumina planorbis & MPN332 & _- & KX215672 \\
\hline Dictyomeridium proponens & MPN359 & JN887415 & JN887403 \\
\hline Dothidea eucalypti & CBS: 143417 & - & MG386106 \\
\hline Dothidea sambuci & DAOM 231303 & AY544739 & AY544681 \\
\hline Elsinoe centrolobii & CBS 222.50 & - & NG 069000 \\
\hline Elsinoe lepagei & CBS 225.50 & - & KX887004 \\
\hline Elsinoe phaseoli & CBS 165.31 & _- & DQ678095 \\
\hline Hortaea werneckii & CBS 708.76 & GU561844 & GU301818 \\
\hline Macroconstrictolumina malaccitula & MPN574 & KM453824 & - \\
\hline Marcelaria cumingii & MPN552 & KM453854 & KM453789 \\
\hline Marcelaria cumingii & UBN137 & LC034284 & - \\
\hline Marcelaria cumingii & RAMK:027993 & LC223105 & LC223104 \\
\hline Marcelaria purpurina & MPN323A & KM453855 & KM453790 \\
\hline Natipusilla decorospora & ILL:AF236-1 & - & NG 060263 \\
\hline Natipusilla limonensis & ILL:AF286-1 & - & NG 060264 \\
\hline Natipusilla naponensis & ILL:AF217-1 & - & NG 060265 \\
\hline Neomicrothyrium siamense & IFRDCC 2194 & _- & JQ036228 \\
\hline Nigrovothelium bullatum & MPN114 & KX215589 & KX215673 \\
\hline Nigrovothelium bullatum & MPN579 & KX215590 & KX215674 \\
\hline Nigrovothelium bullatum & MPN82 & KX215591 & KX215675 \\
\hline Nigrovothelium tropicum & MPN44 & KX215592 & KX215679 \\
\hline Nigrovothelium tropicum & MPN561 & KX215593 & KX215680 \\
\hline Nigrovothelium tropicum & MPN658 & KX215594 & - \\
\hline
\end{tabular}


Table 1 Continued.

\begin{tabular}{|c|c|c|c|}
\hline \multirow{2}{*}{ Taxa } & \multirow{2}{*}{ Strain } & \multicolumn{2}{|c|}{ GenBank Accessions } \\
\hline & & mtSSU & nuLSU \\
\hline Phaeotrichum benjaminii & CBS 541.72 & AY538349 & NG_057709 \\
\hline Polycoccum pulvinatum & Ertz 18114 (BR) & - & КTЗ̄383806 \\
\hline Polycoccum vermicularium & Diederich 17545 & - & KT383808 \\
\hline Polymeridium albocinereum & MPN439 & KM453858 & KM453795 \\
\hline Polymeridium catapastum & MPN358 & KM453859 & JN887402 \\
\hline Polymeridium subcinereum & CBS 130779 & КC592287 & - \\
\hline Polypyrenula sexlocularis & RMG058 & MK503260 & MK503261 \\
\hline Polypyrenula sexlocularis & RMG057 & MK503257 & MK503258 \\
\hline Pseudobogoriella hemisphaeria & Lücking $28641(\mathrm{~F})$ & GU327695 & GU327719 \\
\hline Pseudobogoriella miculiformis & Lücking 28637 (F) & GU327696 & GU327720 \\
\hline Pseudobogoriella minutula & MPN567 & KM453856 & - \\
\hline Pseudopyrenula aff. subgregaria & MPN288 & - & KX215682 \\
\hline Pseudopyrenula diluta & MPN362 & KM453861 & KM453797 \\
\hline Pseudopyrenula diluta & MPN697 & KM453862 & KM453798 \\
\hline Pseudopyrenula endoxanthoides & Lücking 24079 (F) & GU327699 & GU327724 \\
\hline Pseudopyrenula endoxanthoides & MPN573 & KX215595 & - \\
\hline Pseudopyrenula endoxanthoides & MFLU 20-0542 & MZ221618 & MZ206304 \\
\hline Pseudopyrenula subgregaria & MPN391 & KM453863 & KM453799 \\
\hline Pseudopyrenula subgregaria & MPN568 & KX215597 & KX215684 \\
\hline Pseudopyrenula subnudata & MPN293 & KM453865 & KM453801 \\
\hline Roussoella nitidula & MFLUCC 11-0182 & - & KJ474843 \\
\hline Roussoella nitidula & MFLUCC 11-0634 & _- & KJ474842 \\
\hline Roussoella solani & CBS 141288 & - & MH878207 \\
\hline Roussoellopsis tosaensis & KT 1659 & - & AB524625 \\
\hline Sympoventuria capensis & CPC 12839 & - & MK810809 \\
\hline Sympoventuria capensis & CPC 12840 & - & MK810810 \\
\hline Teratosphaeria hortaea & CBS 124156 & - & MH874881 \\
\hline Teratosphaeria tinarooa & CBS 124583 & - & MH874910 \\
\hline Trichodelitschia bisporula & CBS 262.69 & - & MH871039 \\
\hline Trypethelium eluteriae & Lumbsch 19701a (F) & KM453874 & GU327726 \\
\hline Trypethelium eluteriae & MPN563 & KX215599 & KX215686 \\
\hline Trypethelium foveolatum & MPN351 & KM453881 & KM453816 \\
\hline Trypethelium inamoenum & MPN228 & KM453875 & KM453810 \\
\hline Trypethelium platyleucostomum & MPN349 & KM453870 & KM453806 \\
\hline Trypethelium platyleucostomит & MPN350 & KX215602 & KX215688 \\
\hline Trypethelium platystomum & TSL35 & AB759868 & - \\
\hline Trypethelium rubroplatystomum & MPN54 & KM453871 & KM453807 \\
\hline Trypethelium rubroplatystomum & MPN64 & - & KX215689 \\
\hline Trypethelium rubroplatystomum & MPN65C & KX215603 & KX215690 \\
\hline Trypethelium sprengelii & MPN200B & - & KX215691 \\
\hline Trypethelium sprengelii & MPN382 & KM453867 & KM453803 \\
\hline Trypethelium subeluteriae & MPN49C & KM453882 & KM453818 \\
\hline Trypethelium subeluteriae & MPN748 & KX215604 & KX215693 \\
\hline Tumidispora shoreae & MFLUCC 14-0574 & - & KT314074 \\
\hline Venturia albae & CBS 471.61 & - & MK810840 \\
\hline Venturia chlorospora & CBS 466.61 & - & MK810844 \\
\hline Venturia inaequalis & CBS 535.76 & _- & EU035460 \\
\hline Viridothelium tricolor & MPN399 & KM453844 & KM453779 \\
\hline Viridothelium tricolor & MPN646 & KM453845 & KM453780 \\
\hline Viridothelium virens & RAMK:030224 & LC223103 & LC223102 \\
\hline Viridothelium virens & AFTOL-ID 1774 & KT232227 & - \\
\hline Zeloasperisporium eucalyptorum & CBS 124809 & - & NG 057835 \\
\hline Zeloasperisporium pterocarpi & MFLUCC 17-0910 & - & MH763755 \\
\hline Zeloasperisporium searsiae & СРC 25880 & - & NG 059617 \\
\hline
\end{tabular}




\section{Results}

\section{Phylogenetic analyses}

The final alignment comprised 1775 nucleotide positions (mtSSU: 891, nuLSU: 884). The single gene tree topologies and the combined tree topology were compared manually and were found to be congruent. The best scoring ML tree was selected to represent the relationships among the taxa, with the final ML optimization likelihood value of -22431.292969 (Fig. 1). The parameters for the GTR $+\mathrm{I}+\mathrm{G}$ model of combined mtSSU and nuLSU were as follows: estimated base frequencies; $\mathrm{A}=0.305349, \mathrm{C}=0.167827, \mathrm{G}=0.247710, \mathrm{~T}=0.279114$, substitution rates; $\mathrm{AC}$ $=1.088265, \mathrm{AG}=2.892696, \mathrm{AT}=2.180779, \mathrm{CG}=1.068550, \mathrm{CT}=5.897541$ and $\mathrm{GT}=1.0$, proportion of invariable sites $I=0.268570$; gamma distribution shape parameter $\alpha=0.588111$. The ML and Bayesian analyses both resulted in trees with similar topologies. Bayesian posterior probabilities from MCMC were evaluated with final average standard deviation of split frequencies $=0.001817$.

The overall phylogeny matched that of previous studies, including the sister group relationship of Polycoccaceae and Trypetheliaceae and the large, supported, fully lichenized clade ranging from Pseudopyrenula to Astrothelium (Fig. 1). The early diverging lineages representing Alloarthopyrenia, Bogoriella, Constrictolumina, Macroconstrictolumina, Polypyrenula, and Pseudobogoriella (Fig. 1). Arthopyrenia cerasi clustered with strong support with Julella fallaciosa within Trypetheliaceae, whereas A. salicis fell within Roussoellaceae (Pleosporales). The newly generated sequences of Allarthopyrenia italica and Pseudopyrenula endoxanthoides fell with their counterparts representing the same species (Fig. 1).

\section{Ancestral character state analyses}

The supported subclade representing Trypetheliaceae sensu stricto, starting with the genus Pseudopyrenula, exhibits an exclusively lichenized lifestyle, whereas the early diverging clades (Trypetheliaceae sensu lato) show frequent lifestyle switches, including lichenized, non-lichenized saprotrophic, lichenicolous, and borderline lichenized taxa. The sister family, Polycoccaceae, is entirely lichenicolous. Borderline lichens appear in various clades, associated with or nested within either lichenized and saprotrophic lineages. The lichenized Macroconstrictolumina malaccitula formed a sister clade with the borderline lichenized genus Bogoriella. The saprotrophic Arthopyrenia cerasi was strongly supported as sister to the saprotrophic Julella. The borderline lichenized Polypyrenula sexlocularis, was associated with the saprotrophic genus Alloarthopyrenia, while Bogoriella and Pseudobogoriella included weakly (borderline) to more distinctly lichenized taxa. Bayesian MCMC analysis reconstructed the ancestor of Trypetheliaceae as likely lichenized, while Bayes Traits reconstructed the same node as ambiguous and also the basal nodes of the remaining early diverging lineages as ambiguous (Fig. 2). Indeed, while Trypetheliaceae as a whole were reconstructed as de novo lichenized, the reconstruction of gains and losses of lichenization in the early diverging lineages of the family was ambiguous. However, there was a single transition towards stable lichenization associated with a more complex thallus anatomy in the large clade ranging from Pseudopyrenula to Astrothelium, supported by both analyses (Fig. 2).

\section{Taxonomy}

Trypetheliaceae Eschw., Syst. Lich.: 17 (1824)

Type genus: Trypethelium Spreng., Anleit. Kennt. Gew. 3: 350 (1804)

$\equiv$ Arthopyreniaceae Walt. Watson, New Phytol. 28: 107 (1929), syn. nov,

Type genus: Arthopyrenia A. Massal.

Arthopyrenia A. Massal., Ric. Auton. Lich. Crost. (Verona): 165 (1852)

Type: Arthopyrenia cerasi (Schrad.) A. Massal., Ric. Auton. Lich. Crost. (Verona): 167 (1852) 


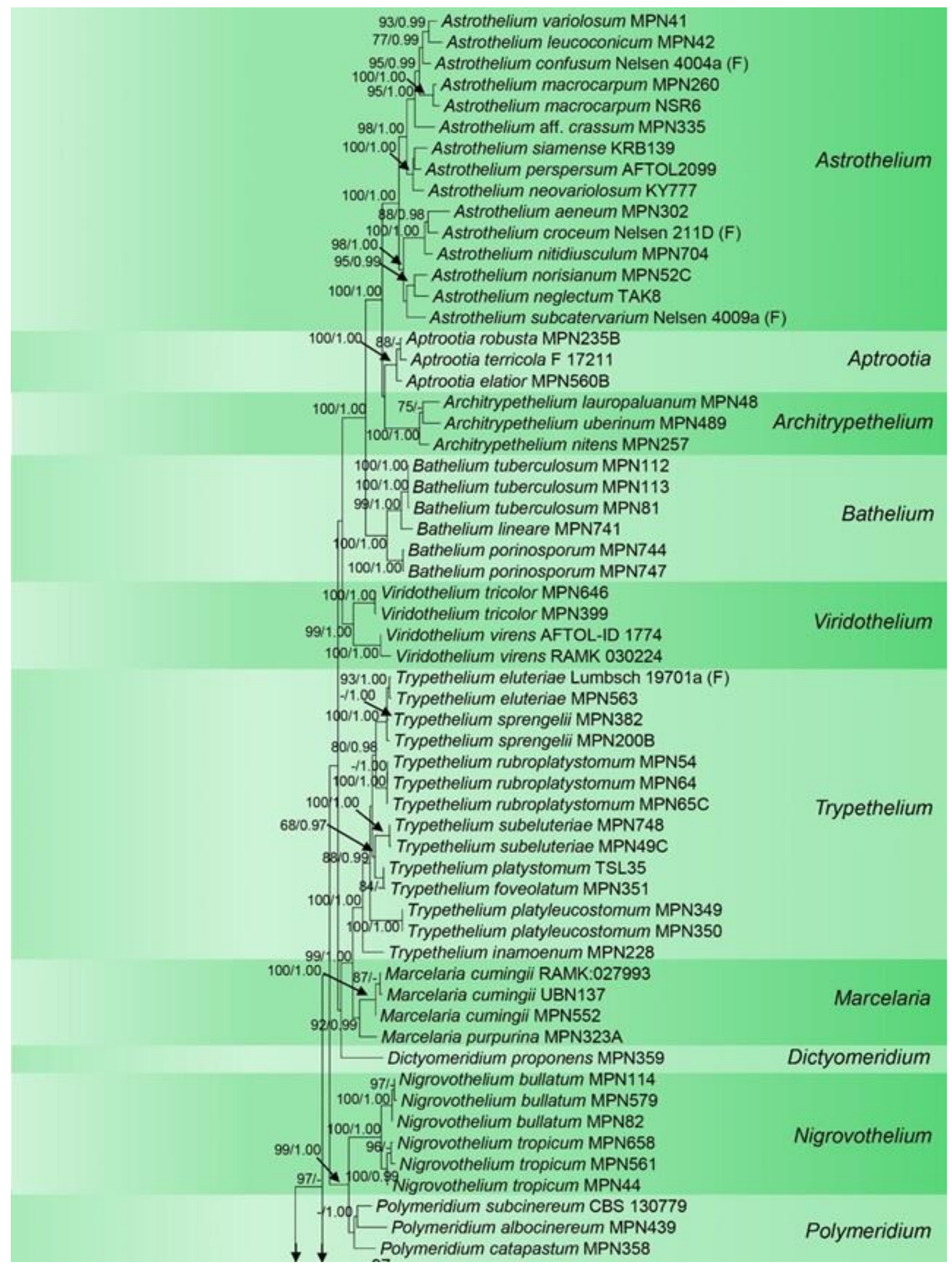

Figure 1 - RAxML tree based on analyses of combined mtSSU and nuLSU partial sequence data. Bootstrap support values for ML equal to or greater than $75 \%$, and Bayesian posterior probabilities (BP) equal to or greater than 0.95 are given as ML/BP above the nodes. The newly generated strains and taxa used in this study are displayed in blue boldface. 


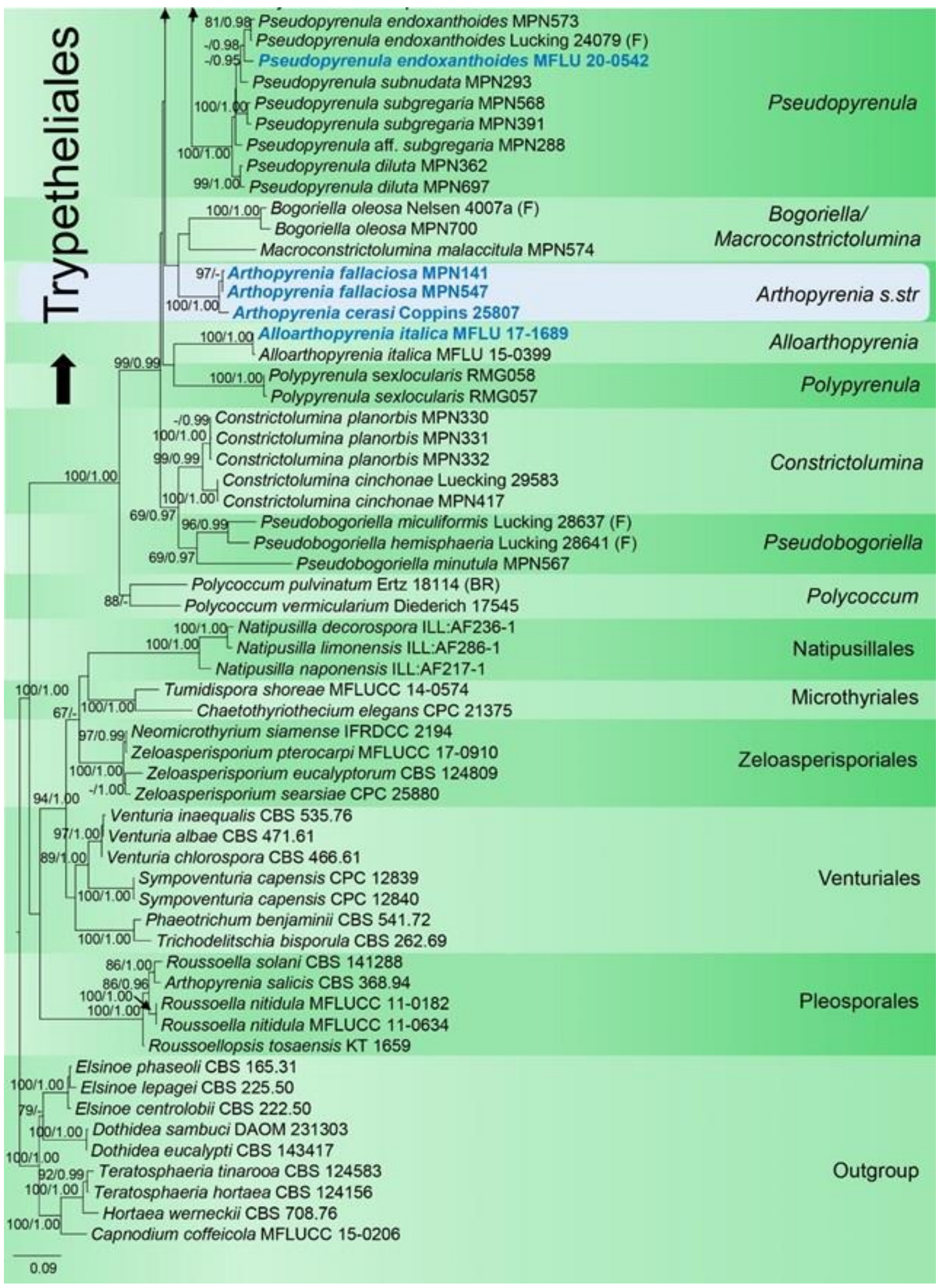

Figure 1 - Continued. 
lichenized

non-lichenized (saprotrophic)

borderline

- lichenicolous

plant pathogenic

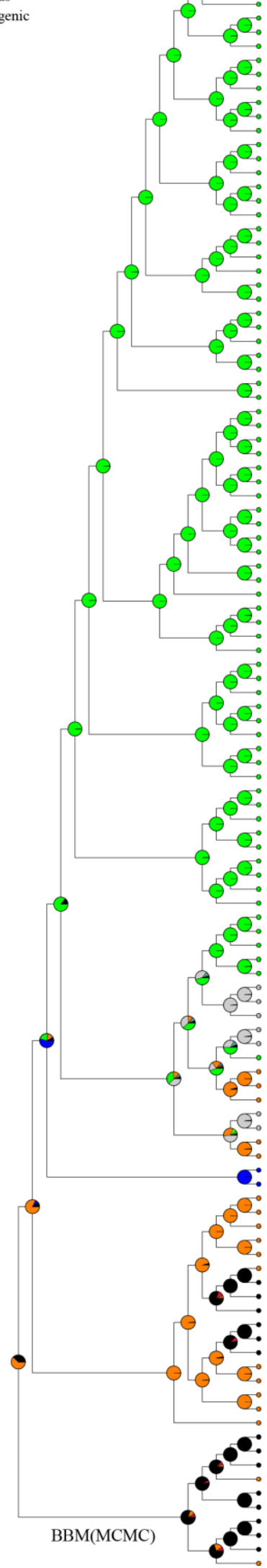

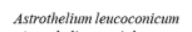
Astrothelium confissum Astrothelinm macrocarpun Istrothelium aff. crassum Astrothelium siamense
Astrothelium perspersum Astrothelinum perspersum Astrothelium aeneum Astrothelium croceum Astrothelium neglectum Astrothelium norisiamun Architrypethelinum uberimum Architypethelinum uberimum
Architrypethelium lauropaluan Anchitrypethelinm niten Aptrootia terricola Aptrootia robusta Aptrootia elatior
Bathelimm tuberculosum Bathelinum tuberculosum Bathelium tuberculosum Bathelinum lineare Buthelimm porinosporne Bathelium porinospornm
Viridothelium virens iridothelimm virens Viridothelium tricolor
Viridothelinum tricolor Viridothelinum tricolor Dictyomeridium proponen Dictyomeridium proponen Trppethelinum eluteriae
Trypethelium eluteriae Thpethelium sprengelii Typethelium sprengelii Trypethelium rubroplatysto Trypethelium rubroplatystomum Typethelium rubroplatsstomm
Typethelitum platystommm Typethelim subeluterie rypethelium platylencostomum Trypethelinum platyleucostomum
Trypethelium inamoermum Inpethelium inamoemum
Marcelaria cumingii Marcelaria cumingii Narcelaria purpuring grovothelitum bullatur Nigrovothelium bullatum Nigrovothelium bullatum
Nigrovothelium tropicue Nigrovothelinum tropicum
Nigrovothelium tropicum Nigrovothelium tropicul
Nigrovothelium tropicue Polyneridiun subcinereun Polymeridium subcinereum
Polymeriditum albocinereum Polymeridium catapastum Psendopyrenula aimua
Psendiopyrenula diluta Pseudopyremula aff subgregaria
Pseudopyremula subgregaria Pseudopyremula subgregaria Psendopyremula endoxanthoides Psendopyremila endoxanthoides
Psendopyremula endoxanthoides

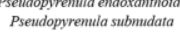
Constrictolumina planorb Constrictolumina planorb Constrictolumina cinchonae
Constrictolumina cinchonae seudobogoriella miculifor Pseudobogoriella mimutula Bogoriella oleos
Bogoriella oleos Macroconstrictolumina mala
Arthopyreniafallaciosa Arthopyreniafallacios Arthopyrenia cerasi
Polypyremula sexloculari Polypyremula sexlocularis Alloarthopyrenia italica Alloarthopyremia italica Polycoccum vermicularium
Polycoccum pulvinatum Polycoccum pulvinatum Natipusill a decorospor
Natipusilla limonensi. Natipusilla naponensis Tumidispora shoreae Chaetothyriothecium eleg Eeloasperisporium pteroc Zeloasperisporium searssiae Venturia inaequals
Venturia albae Venturia chlorospora sympoventuria capensis T. Trichodelitschia bisporila
Phaeotrichum benjaminii Arthopyrenia salicis Elsinoe phaseoli
Elsinoe lepagei Elsimoe lepagei Elsinoe centrolobii
(yriangium hispanicu Dothidea encalyp Teratosphaeria hortaed Hortaea werneckii

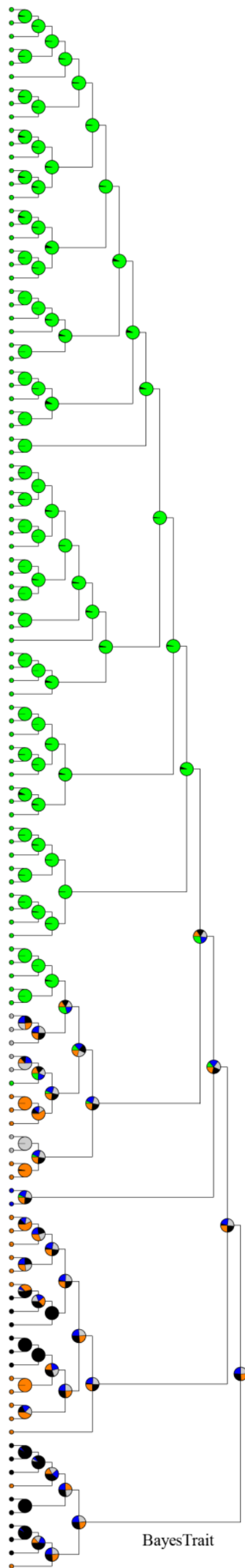

Figure 2 - Ancestral character state analyses using Bayesian MCMC (left) and Bayes Traits (right). Color symbols indicates: green - lichenized, orange - non-lichenized saprotrophic, grey borderline lichen, blue - lichenicolous, black - plant pathogens 
Notes - Arthopyrenia was introduced by Massalongo (1852), originally including 13 species but without indicating a type species. Among these, only Arthopyrenia analepta, A. cerasi, and A. salicis have remained in this genus over time, while the remaining species have been transferred to different genera and families. Massalongo (1854) himself transferred two Arthopyrenia species to the newly established Acrocordia. Arthopyrenia was first lectotypified by Fink (1910) with A. rhyponta, which subsequently became a member of Naetrocymbe. When the generic name Arthopyrenia was conserved, A. cerasi was chosen as the conserved type (Gams 1999). The taxonomy of Arthopyrenia was studied by Jatta (1911), Vainio (1921), Harris (1973, 1975), Tucker \& Harris (1980) and Coppins (1988). Harris (1975) established Naetrocymbaceae to accommodate the single genus Naetrocymbe, transferring a few non-lichenized species to that genus and retaining Arthopyrenia, Julella and Mycomicrothelia within Arthopyreniaceae. Naetrocymbe species were thereby characterized by short-celled paraphyses with refractive bodies near the septa, obpyriform asci with a distinctive apical region, lacking a nasse, and short, rod-shaped microconidia. Aptroot $(1998,2002)$ emphasized the branched pseudoparaphyses as an important character. Nonetheless, many authors accepted Harris's classification (Eriksson et al. 2003, Nelsen et al. 2009, 2011, 2014, Hyde et al. 2013, Wijayawardene et al. 2017, 2018, 2020, Hongsanan et al. 2020a). Harris (1995) pointed out the close relationship between Julella and Arthopyrenia, the only difference being ascospore septation, a notion supported by other authors (Aptroot et al. 2008, Nelsen et al. 2011). The first sequenced non-lichenized species thus far retained within Arthopyrenia, A. salicis, was subsequently resolved within Pleosporales (Pinnoi et al. 2007, Zhang et al. 2008, Sar et al. 2009, Nelsen et al. 2011, 2014, Liu et al. 2014). In contrast, tropical lichenized species nested within Trypetheliales, rendering Arthopyrenia polyphyletic (Nelsen et al. 2011). Our results now show that even non-lichenized species of Arthopyrenia form a polyphyletic assembly and that Arthopyrenia sensu stricto is to be placed within Trypetheliaceae.

Arthopyrenia cerasi (Schrad.) A. Massal., Ric. auton. lich. crost. (Verona): 167 (1852)

Fig. 3

Index Fungorum number: IF 377023; Facesoffungi number: FoF 09867

Description (adapted from Coppins \& Orange 2009 and Hyde et al. 2013 and including assessment of sequenced material). Thallus inconspicuous or slightly bleaching the bark, endophloeodal. Photobiont absent. Sexual morph: Ascomata perithecial, c. 300-500 $\mu \mathrm{m}$ diam., black, rounded to often ellipsoid, somewhat adnate, ostiolate. Ostiole distinct, centrally located. Involucrellum dark brown, $\mathrm{K}+$ greenish. Exciple light brown. Pseudoparaphyses slender, anastomosing, 1.5-2 $\mu \mathrm{m}$. Asci $80-85 \mu \mathrm{m}$, 8-spored, bitunicate, cylindrical-clavate. Ascospores 17$22 \times 5-7 \mu \mathrm{m}$, irregularly biseriate, hyaline, clavate to obovoid, rounded at the apex when mature, constricted at each septum, 3 -septate, with a gelatinous sheath c. $2 \mu \mathrm{m}$ thick in K. Asexual morph: Pycnidia 80-120 $\mu \mathrm{m}$, with either macro- or microconidia. Macroconidia 11-13 $\times 2-3 \mu \mathrm{m}$, oblong, hyaline, 3-septate. Microconidia 9-14 × $0.8 \mu \mathrm{m}$.

Material examined - Great Britain, Scotland, VC82, East Lothian, Stenton, Cow Cleugh Burn, Grid NT617718, $160 \mathrm{~m}$ elev., on Corylus in small valley woodland, 18 v 2020, B.J. \& A.M. Coppins 25807 (BR, E).

Notes - Arthopyrenia cerasi is characterized by a non-lichenized thallus, perithecial ascomata with a brown, $\mathrm{K}+$ greenish involucrellum, anastomosing pseudoparaphyses, cylindrical asci with hyaline 3-septate ascospores and conidiomata producing frequently 3-septate macroconidia (Coppins 1988). This species often grows on Corylus spp. and is distributed in Europe (Coppins \& Orange 2009).

Arthopyrenia fallaciosa (Stizenb. ex Arnold) Thiyagaraja, Ertz, Lücking, Coppins and K.D. Hyde comb. nov.

Index Fungorum number: IF 558410; Facesoffungi number: FoF 09868

Basionym: Polyblastia fallaciosa Stizenb. ex Arnold, Flora, Regensburg 46: 604 (1863).

Obligate synonyms: Pyrenula fallaciosa (Stizenb. ex Arnold) Willey, Enum. Lich. New Bedford: 39 (1892); Verrucaria fallaciosa (Stizenb. ex Arnold) Nyl., Lich. Envir. Paris: 127 
(1896); Mycoglaena fallaciosa (Stizenb. ex Arnold) Vain., Acta Soc. Fauna Flora fenn. 49(no. 2): 166 (1921); Polyblastiopsis fallaciosa (Stizenb. ex Arnold) Zahlbr., Cat. Lich. Univers. 1: 348 (1922); Julella fallaciosa (Stizenb. ex Arnold) R.C. Harris, in Egan, Bryologist 90(2): 163 (1987).

Notes - Our updated phylogeny resulted in a close relationship between Arthopyrenia cerasi and Julella fallaciosa, to the point that the latter is to be placed within Arthopyrenia. This is supported by the fact that, apart from the transversely septate vs. muriform ascospores, J. fallaciosa and related species are morphologically and anatomically very similar to Arthopyrenia sensu stricto and including these in Arthopyrenia has been suggested even prior to molecular studies (Harris 1995). Lücking et al. (in Hongsanan et al. 2020b) provided a discussion on the taxonomy of Julella, pointing out that at least two groups can be distinguished: species related to the type, J. buxi, and species related to J. lactea, for which the genus name Polyblastiopsis is available. Julella fallaciosa arguably belongs to the latter group, which implies that Polyblastiopsis would be a synonym of Arthopyrenia. However, more species currently classified within Julella need to be sequenced to clarify this. Given that Polyblastiopsis is younger than Arthopyrenia, those species clustering with A. cerasi can be safely combined into the latter genus.
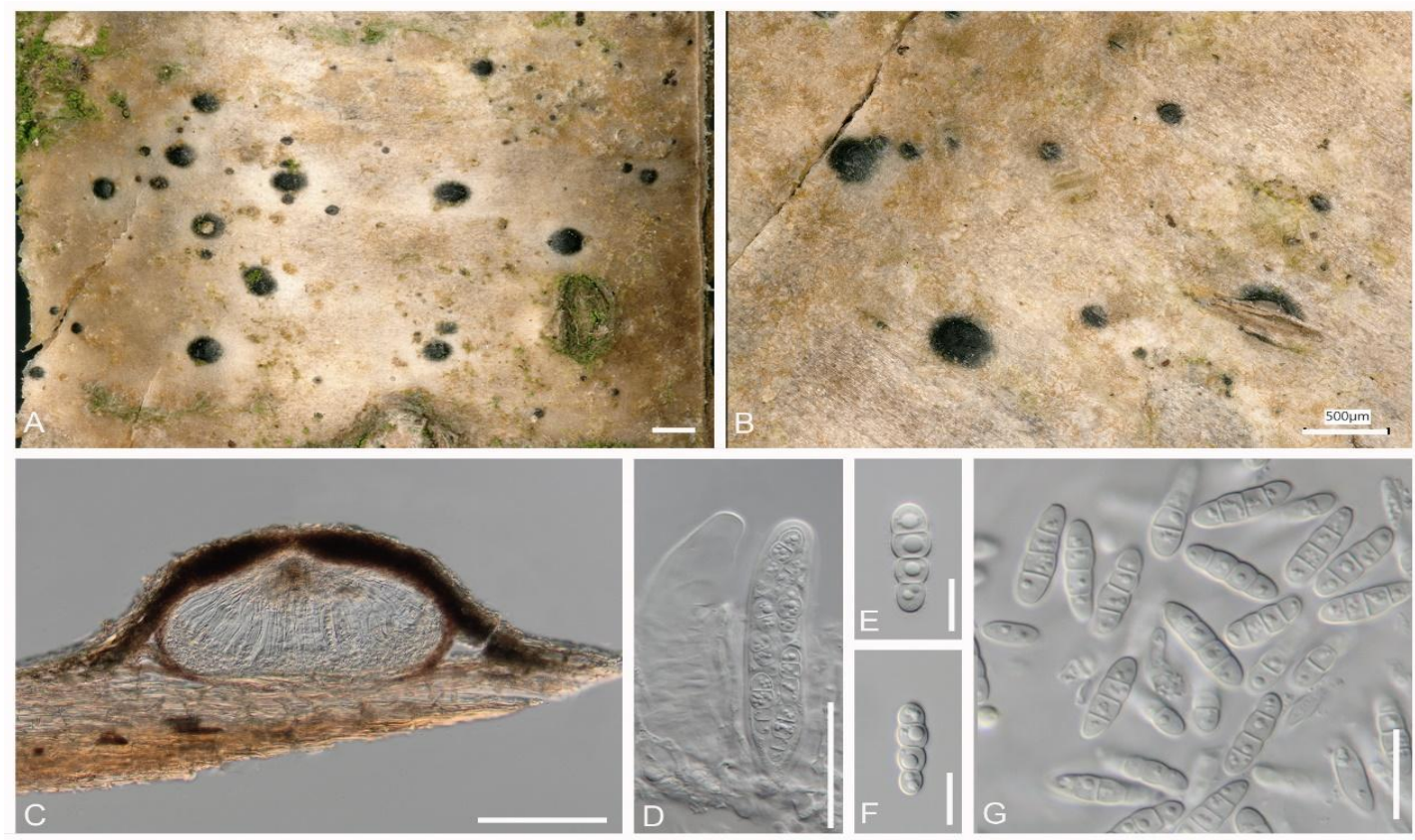

Figure 3 - Arthopyrenia cerasi (Coppins 25807). A, B Ascomata and conidiomata on bark. $\mathrm{C}$ Cross section of ascoma in water. D Asci in water. E, F Ascospores in water. G Macroconidia in water. Scale bars: $\mathrm{B}=500 \mu \mathrm{m}, \mathrm{C}=100 \mu \mathrm{m}, \mathrm{D}=30 \mu \mathrm{m}, \mathrm{E}-\mathrm{G}=10 \mu \mathrm{m}$

Alloarthopyrenia italica Phukhams., Camporesi, Ariyaw. \& K.D. Hyde, Fungal Diversity 80: 135 (2016)

= Arthopyrenia cinereopruinosa auct., non Schaerer (1836).

Index Fungorum number: IF 552237, Facesoffungi number: FoF 02380

Non-lichenized on bark. Thallus inconspicuous, whitish grey, pruinose, corticolous, crustose, epiphloeodal. Prothallus absent. Photobiont not detected. Sexual morph: Ascomata perithecial, approximately 135-165 $\mu \mathrm{m}$ high $\times 300-375 \mu \mathrm{m}$ diam., black, circular to ellipsoidal, slightly erumpent, somewhat adnate, ostiolate. Ostiole distinct, centrally located, filled with periphyses. Involucrellum dark brown, $\mathrm{K}+$ slightly greenish. Exciple 40-70 $\mu \mathrm{m}$, light brown. Hamathecium 75$110 \mu \mathrm{m}$ high $\times 195-210 \mu \mathrm{m}$ diam. Pseudoparaphyses robust, \pm distantly branched, numerous and anastomosed. Asci 55-65 × 13-20 $\mu \mathrm{m}(\overline{\mathrm{x}}=60 \times 16.5 \mu \mathrm{m}, \mathrm{n}=40), 8$-spored, bitunicate, cylindrical, tholus thickened, ocular chamber up to $2-3 \mu \mathrm{m}$, apically rounded, poorly developed stipe, inversely funnel-shaped ocular chamber. Ascospores 18-22 (24) $\times 8-12 \mu \mathrm{m}(\overline{\mathrm{x}}=20 \times 10 \mu \mathrm{m}, \mathrm{n}=40)$, multi- 
seriate, hyaline, clavate to obovoid, 1-septate, rounded at the apex when mature, strongly constricted at the septa, upper cell wider than lower cell, gelatinous sheath distinct, 1-3 $\mu \mathrm{m}$ thick. Asexual morph: unknown.

Material examined - Italy, Province of Forlì-Cesena, near Monte Mirabello - Predappio,16 September 2017, on living Fraxinus ornus, Erio Camporesi (MFLU 17-1689).

Chemistry - Thallus I-, Ascomatal gel I-, K-. Asci I-, K-. Ascospores I-, K-.

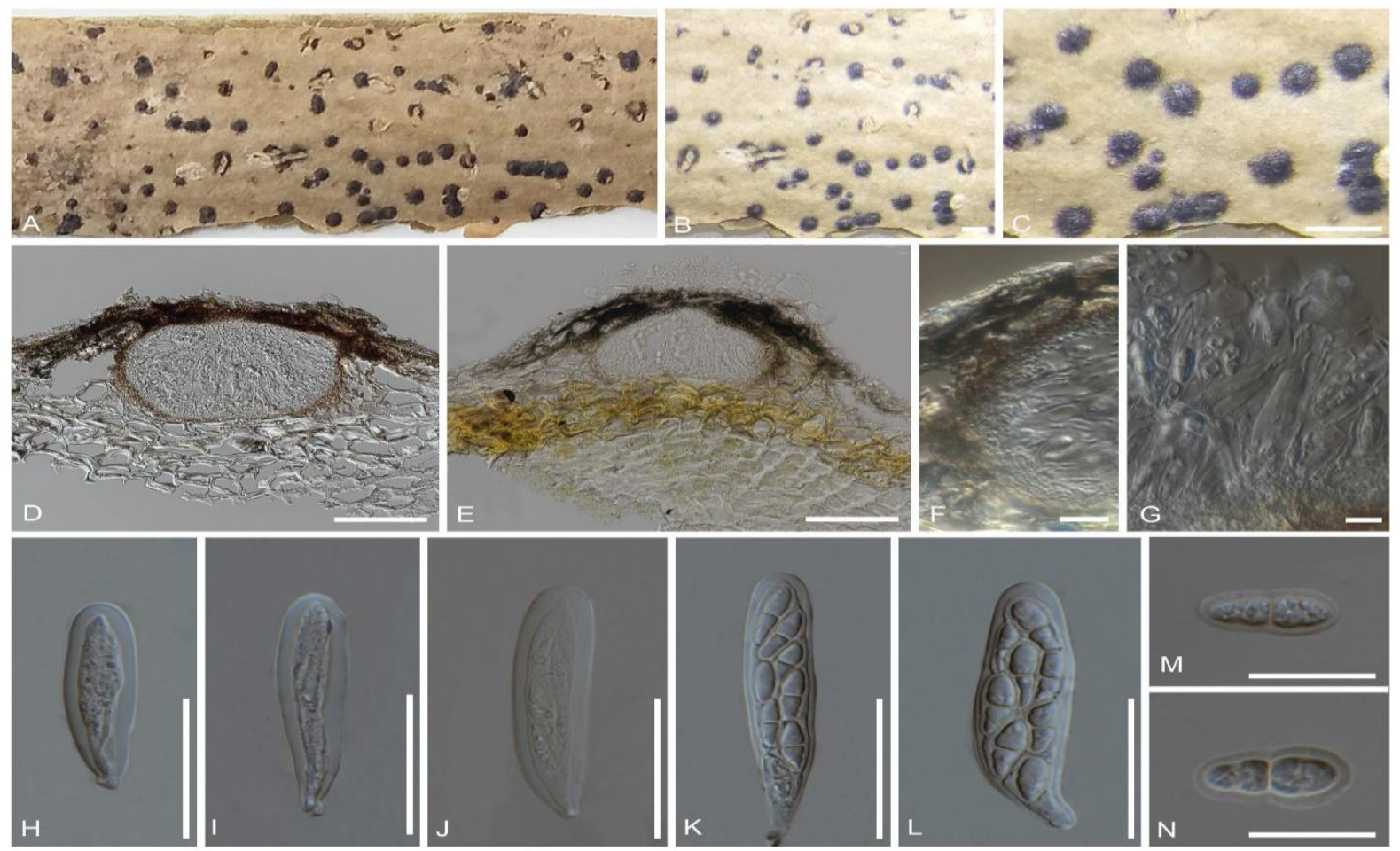

Figure 4 - Alloarthopyrenia italica (MFLU 17-1689). A- C, Ascomata on bark. D Cross section of ascoma in water. E Cross section of ascoma in 5\% KOH. F Peridium. G Pseudoparaphyses. $\mathrm{H}-\mathrm{L}$ Asci in tap water. M, N Ascospores in tap water. Scale bars: $\mathrm{B}-\mathrm{C}=500 \mu \mathrm{m}, \mathrm{D}, \mathrm{E}=100 \mu \mathrm{m}$, $\mathrm{F}=50 \mu \mathrm{m}, \mathrm{G}=10 \mu \mathrm{m}, \mathrm{H}-\mathrm{L},=30 \mu \mathrm{m}, \mathrm{M}, \mathrm{N}=20 \mu \mathrm{m}$.

Notes - Alloarthopyrenia was introduced to accommodate a single species, A. italica (Hyde et al. 2016). The species was collected from living bark of branches of Fraxinus ornus in Italy (Hyde et al. 2016). The species shares morphological characteristics with other non-lichenized Arthopyrenia species (Hyde et al. 2016). Here we provide additional data for Alloarthopyrenia italica from material that was also collected in Italy. The resemblance of this species to Arthopyrenia cinereopruinosa (Schaer.) A. Massal. is striking and a more detailed comparison of these two taxa is in order. Alloarthopyrenia italica was originally separated from Arthopyrenia cinereopruinosa based on larger ascomata and 3-septate ascospores. The presumed type material of A. cinereopruinosa (basionym: Verrucaria cinereopruinosa) in H (Switzerland, Schaerer s.n., HNYL 6684) has a leprose thallus and ascomata with 3-septate ascospores that somewhat resemble Chrysothrix caesia [https://plants.jstor.org/stable/viewer/10.5555/al.ap.specimen.h9507588]. This material cannot be original, as it was only published in Schaerer's exsiccate, Enum. Critic. Lich. $\begin{array}{lllll}\text { Europ., } & \text { as } & \text { number } & 243, & \text { in }\end{array}$ [https://www.biodiversitylibrary.org/item/109124\#page/287/mode/1up], when the species was validly established 14 years earlier (Schaerer 1836). In the latter protologue, Schaerer (1836) listed four specimens, all as names but not representing the types of these names. Three of these were associated with the name Verrucaria cinereopruinosa and one with the variety galactina, based on Arthonia punctiformis var. galactina. The first specimen, originally identified as Verrucaria stigmatella by Schlechtendahl, is deposited in $\mathrm{G}$ and available on JSTOR 
[https://plants.jstor.org/stable/viewer/10.5555/al.ap.specimen.g00290379]. Verrucaria stigmatella was described as non-leprose thallus and perithecia that are almost pseudostromatic, with clusters of up to 10 small perithecia, whereas typical A. cinereopruinosa as currently circumscribed has single to sometimes grouped, larger perithecia not in such clusters. It does not correspond to the modern concept of $A$. cinereopruinosa but rather corresponds to Naetrocymbe punctiformis. It therefore appears that what has usually been regarded A. cinereopruinosa (e.g. Harris 1975, Coppins 1988) [https://fungi.myspecies.info/all-fungi/arthopyrenia-cinereopruinosa] is not conspecific with the type of that name but corresponds to what is now named Alloarthopyrenia italica.

Pseudopyrenula endoxanthoides Vain., Hedwigia 46: 180 (1907)

Fig. 5

Index Fungorum number: IF 402510; Facesoffungi number: FoF 09869

Lichenized on bark. Thallus present, whitish, non-pruinose, corticolous, crustose, epiphloeodal. Prothallus present. Photobiont trentepohlioid. Sexual morph: Ascomata perithecial, 245-315 $\mu \mathrm{m}$ high $\times 375-500 \mu \mathrm{m}$ diam., black, rounded to ellipsoid, slightly erumpent, somewhat adnate, ostiolate. Ostiole distinct, centrally located. Involucrellum $20-45 \mu \mathrm{m}$ thick, light brown, no color change in KOH. Exciple 15-40 $\mu \mathrm{m}$, dark brown. Hamathecium 180-230 $\mu \mathrm{m}$ high $\times 245-295$ $\mu \mathrm{m}$ diam, occasionally yellow. Pseudoparaphyses robust, \pm distantly branched, numerous and anastomosed. Asci 65-75 $\times 10-15 \mu \mathrm{m}(\overline{\mathrm{x}}=70 \times 12.5 \mu \mathrm{m}, \mathrm{n}=40), 8$-spored, bitunicate, cylindrical, tholus thickened, ocular chamber inversely funnel-shaped, up to 4-5 $\mu \mathrm{m}$, apically rounded, with a well-developed stipe. Ascospores $17-20 \times 4-10 \mu \mathrm{m}(\overline{\mathrm{x}}=18.5 \times 7 \mu \mathrm{m}, \mathrm{n}=40)$, irregularly biseriate, hyaline to pale yellow, clavate to obovoid, rounded at the apex when mature, 1-3-septate, gelatinous sheath distinct, 1-2 $\mu \mathrm{m}$ thick. Asexual morph: unknown.
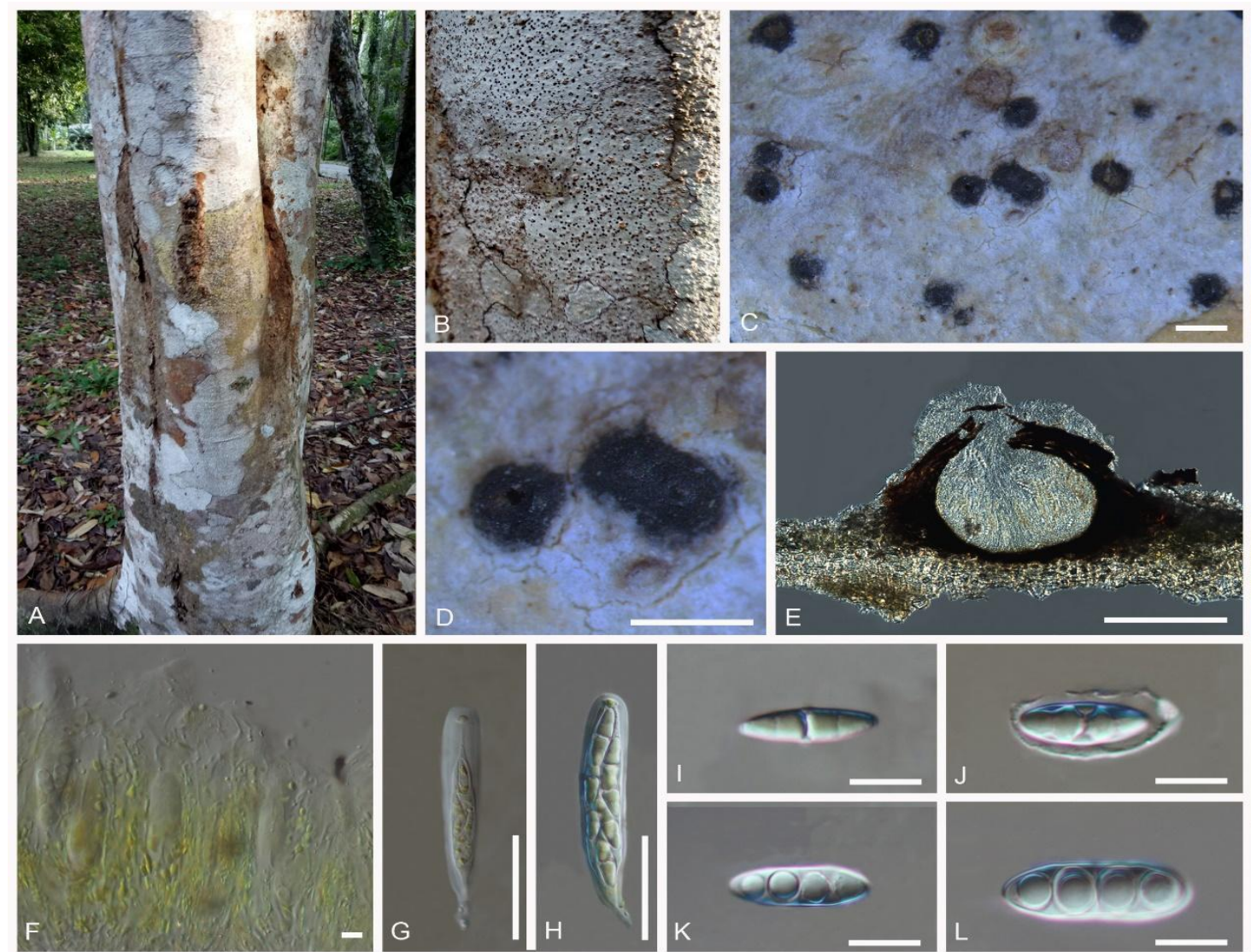

Figure 5 - Pseudopyrenula endoxanthoides (MFLU 20-0542). A-D Ascomata on bark. E Cross section of ascoma in water. F Pseudoparaphyses. G, H Asci in tap water. I-L Ascospores in tap water. Scale bars: $\mathrm{C}, \mathrm{D}=500 \mu \mathrm{m}, \mathrm{E}=200 \mu \mathrm{m}, \mathrm{F}=10 \mu \mathrm{m}, \mathrm{G}, \mathrm{H}=30 \mu \mathrm{m}, \mathrm{I}-\mathrm{L}=10 \mu \mathrm{m}$. 
Material examined - Thailand, Hat Yai, on unidentified tree, 12 May 2018, V. Thiyagaraja, TV106 (MFLU 20-0542)

Notes - Pseudopyrenula was introduced by Müller (1883), with P. diluta as the type. Taxa in this genus are characterized by an ecorticate thallus and hyaline, transversely septate, astrothelioid ascospores with diamond-shaped lumina (Aptroot \& Lücking 2016). The yellow oil droplets in the ascospore lumina and/or the hamathecium characterizes several species. This genus comprises 12 species (Species Fungorum 2021) and mainly occurs in tropical areas (Aptroot \& Lücking 2016). In this study, Pseudopyrenula was recovered as a monophyletic clade in the phylogenetic analyses, which concurs with previous studies (Nelsen et al. 2014, Lücking et al. 2016b), with an additional new sequenced terminal corresponding to Pseudopyrenula endoxanthoides. The latter is distributed in the Eastern Palaeotropics including Thailand.

\section{Discussion}

Lichenization is an important phenomenon in the evolution of fungi (Lipnicki 2015, Lücking et al. 2017), particularly within the Ascomycota, where it has been repeatedly gained and lost (da Silva Cáceres et al. 2020, Nelsen et al. 2020, Thiyagaraja et al. 2020, 2021). Lichenized lineages are found in several classes within Ascomycota, including at least five major lineages within Dothideomycetes (Nelsen et al. 2009, 2011, Schoch et al. 2009). Within the latter, Trypetheliaceae is the most speciose and one of the nutritionally most diverse lineage that predominantly comprise lichenized taxa. In this family, the early diverging lineages chiefly represent non-lichenized or weakly lichenized taxa, including species of Alloarthopyrenia, Arthopyrenia, Bogoriella, Constrictolumina, Polypyrenula, and Pseudobogoriella, whereas the main clade is exclusively lichenized, with an anatomically more complex thallus organization (Nelsen et al. 2014, Hyde et al. 2016). While the diversity of nutritional strategies in Trypetheliaceae is comparable to that of Arthoniaceae and Stictidaceae, the underlying evolutionary histories are quite different. Thus, Stictidaceae is deeply nested within the predominantly lichenized Lecanoromycetes, clearly indicating its non-lichenized lineages as secondarily non-lichenized (Thiyagaraja et al. 2021). The origin of lichenization in Arthoniales is less clear, but also here the non-lichenized lineages within Arthonia sensu lato appear to be secondarily delichenized (Thiyagaraja et al. 2020). In contrast, Trypetheliaceae as a whole can be reconstructed as a de-novo lichenization event within Ascomycota, showing a clear progression from saprotrophic or weakly lichenized, early diverging lineages that apparently experimented with lichenization to a larger derived clade, including most species of the family, with stable lichenization and a complex thallus anatomy associated with this lifestyle (Lücking et al. 2016b).

The family status of Arthopyreniaceae and its phylogenetic relationships have been debated for a long time, owing to lack of molecular data. Based on the morphological characteristics of the type species, it was hitherto placed in Pleosporales, whereas non-type species with available molecular data clustered elsewhere (Nelsen et al. 2011, 2014, Hyde et al. 2013, Liu et al. 2014). Arthopyreniaceae presumably differs from Trypetheliaceae in several important characteristics, such as cellular pseudoparaphyses, broadly clavate asci and a non-refractive ocular chamber, whereas Trypetheliaceae possess trabeculate pseudoparaphyses, obclavate to cylindrical asci with a refractive ring with a wide ocular chamber (Hyde et al. 2013, Nelsen et al. 2014). However, these differences become diffuse when considering the anatomical variation of early diverging lineages now included in Trypetheliaceae, and so the now confirmed placement of Arthopyrenia sensu stricto within that family is not entirely surprising. The close relationship between A. cerasi and Julella fallaciosa sheds new light on the potential status of species currently included in Julella sensu lato. Following Barr (1985), Harris (1995) included Julella within Arthopyreniaceae, whereas before it was classified within various families, such as Amphisphaeriaceae (Lindau 1897), Pleosporaceae (von Arx \& Müller 1975), and Thelenellaceae (Cannon \& Kirk 2007). Presently only Julella fallaciosa has been sequenced and our results show that the muriform ascospores in this taxon have no taxonomic value at the genus level, as already suggested elsewhere (Harris 1995, 
Aptroot et al. 2008) and also shown for other genera in Trypetheliaceae (Nelsen et al. 2011, Hongsanan et al. 2020b).

Arthopyrenia salicis, described by Massalongo (1852), has been recorded as lichenized, nonlichenized and sometimes both within the same population. A detailed morphological description was provided by Coppins \& Orange (2009), who included several important diagnostic features such as ascomata often with a depressed ostiole lined with periphysoids, an involucrellum reacting brown in K, and the absence of interascal hyphae. Arthopyrenia salicis thus differs from Arthopyrenia sensu stricto in various important characters (Nelsen et al. 2009, 2011, Hyde et al. 2016), which is in accordance with its different phylogenetic placement (Nelsen et al. 2009, 2011, Liu et al. 2014). Molecular sequence data support its close relationship to Roussoella and Roussoellopsis (Liu et al. 2014, Nelsen et al. 2014). However, Arthopyrenia salicis differs from both genera in the absence of paraphysoids and in the oblong to fusiform, hyaline spores lacking striations (Hyde et al. 2016). Hence its exact generic position remains unclear. Unfortunately, the available sequence data for this species are also partly inconsistent, requiring studies of additional specimens before a conclusion on its genus-level taxonomy can be drawn.

\section{Acknowledgements}

We are grateful to grants from the Thailand Research Fund ("The future of specialist fungi in a changing climate: baseline data for generalist and specialist fungi associated with ants, Rhododendron species and Dracaena species DBG6080013" and "Impact of climate change on fungal diversity and biogeography in the Greater Mekong Sub-region RDG6130001") for funding this research. Kevin D. Hyde thanks Chiang Mai University for the Award of Visiting Professor. S.C. Karunarathna would like to thank CAS President's International Fellowship Initiative (PIFI) young staff under the grant number: 2020FYC0002 and the National Science Foundation of China (NSFC, project code 31851110759). Dhanushka Wanasinghe would like to thank CAS President's International Fellowship Initiative (PIFI) for funding his postdoctoral research (number 2021PC0008), the National Science Foundation of China and the Chinese Academy of Sciences for financial support under the following grants: 41761144055, 41771063 and Y4ZK111B01. We thank Thailand Science Research and Innovation (TSRI) grant "Macrofungi diversity research from the Lancang-Mekong Watershed and Surrounding areas (grant no. DBG6280009). We also thank

Udeni Jayalal, Nalin Wijayawardene and Erio Camporesi for their precious help during this research.

\section{References}

Aptroot A. 1998 - Aspects of the integration of the taxonomy of lichenized and nonlichenized pyrenocarpous ascomycetes. Lichenologist 30, 501-514.

Aptroot A. 2002 - Arthopyrenia. In Lichen Flora of the Greater Sonoran Desert Region. Volume I (T. H. Nash III, B. D. Ryan, C. Gries \& F. Bungartz, eds), 103-106. Tempe: Lichens Unlimited.

Aptroot A, Lücking R. 2016 - A revisionary synopsis of the Trypetheliaceae (Ascomycota: Trypetheliales). Lichenologist 48, 763-982.

Aptroot A, Ertz D, Salazar JAE, Gueidan C et al. 2016 - Forty-six new species of Trypetheliaceae from the tropics. Lichenologist 48, 609-638.

Aptroot A, Lücking R, Sipman HJM, Umaña L, Chaves JL. 2008 - Pyrenocarpous lichens with bitunicate asci: a first assessment of the lichen biodiversity inventory of Costa Rica. Bibliotheca Lichenologica 97, 1-162.

Barr ME. 1985 - On Julella, Delacourea, and Decaisnella, three dictyosporous genera described by JH Fabre. In Sydowia: Annales mycologici.

Cannon PF, Kirk PM. 2007 - Fungal families of the world. CABI Bioscience, Wallingford

Coppins BJ. 1988 - Notes on the genus Arthopyrenia in the British Isles. Lichenologist 20(4), 305325. 
Coppins BJ, Aptroot A. 2008 - New species and combinations in the lichens of the British Isles. Lichenologist 40(5), 363.

Coppins BJ, Orange A. 2009 - Arthopyrenia A. Massal. In: Smith CW, Aptroot A, Coppins BJ, Fletcher A, Gilbert OL, James PW, Wolseley PA. (eds), The Lichens of Great Britain and Ireland. London: British Lichen Society 171-176.

da Silva Cáceres ME, Lücking R, Schumm F, Aptroot A. 2020 - A lichenized family yields another renegade lineage: Papilionovela albothallina is the first non-lichenized, saprobic member of Graphidaceae subfam. Graphidoideae. The Bryologist 123(2), 144-154.

Eriksson OE, Baral HO, Currah RS, Hansen K et al. 2003 - Outline of Ascomycota 2003. Myconet 9(1), 1-189.

Ertz D, Guzow-Krzemińska B, Thor G, Łubek A et al. 2018 - Photobiont switching causes changes in the reproduction strategy and phenotypic dimorphism in the Arthoniomycetes. Scientific Reports 8, 4952.

Ertz D, Tehler A, Irestedt M, Frisch A et al. 2015 - A large-scale phylogenetic revision of Roccellaceae (Arthoniales) reveals eight new genera. Fungal Diversity 7, 31-53.

Fink B. 1910 - The lichens of Minnesota. Contributions from the U.S. National Museum. 14, 1269

Gams W. 1999 - Report of the committee for fungi: 8. Taxon 48, 807-810

Glez-Peña D, Gómez-Blanco D, Reboiro-Jato M, Fdez-Riverola F et al. 2010 - ALTER: programoriented conversion of DNA and protein alignments. Nucleic Acids Research 38, 14-18.

Gueidan C, Villaseñor CR, De Hoog GS, Gorbushina AA et al. 2008 - A rock-inhabiting ancestor for mutualistic and pathogen-rich fungal lineages. Studies in Mycology 61, 111-119.

Hall TA. 1999 - BioEdit: A user-friendly biological sequence alignment editor and analysis program for Windows 95/98/NT. Nucleic Acids Symposium Series 41, 95-98. London, England: Information Retrieval.

Harris RC. 1973 - The corticolous pyrenolichens of the Great Lakes Region. Michigan Botanist 12, 3-68.

Harris RC. 1975 - A taxonomic revision of the genus Arthopyrenia Massal. s. lat. (Ascomycetes) in North America. PhD thesis, University of Michigan.

Harris RC. 1995 - More Florida Lichens including the 10ф Tour of the Pyrenolichens. Publ. by author, Bronx, New York. 192

Harris R, Tripp E. 2013 - Arthopyrenia betulicola (Arthopyreniaceae, Dothidiomycetes), an unusual new lichenized fungus from high elevations of the southern appalachian mountains. Aliso 31, 77-81.

Hongsanan S, Hyde KD, Phookamsak R, Wanasinghe DN et al. 2020a - Refined families of Dothideomycetes: Dothideomycetidae and Pleosporomycetidae. Mycosphere 11, 1553-2107.

Hongsanan S, Hyde KD, Phookamsak R, Wanasinghe DN et al. 2020b - Refined families of Dothideomycetes: Orders and families incertae sedis in Dothideomycetes. Fungal Diversity 105, 17-318.

Huelsenbeck JP, Ronquist F. 2001 - MRBAYES: Bayesian inference of phylogenetic trees. Bioinformatics 17, 754-755.

Hyde KD, Hongsanan S, Jeewon R, Bhat DJ et al. 2016 - Fungal diversity notes 367-490: taxonomic and phylogenetic contributions to fungal taxa. Fungal Diversity 80, 1-270.

Hyde KD, Jones EG, Liu JK, Ariyawansa H et al. 2013 - Families of Dothideomycetes. Fungal Diversity 63(1), 1-313.

Index Fungorum. 2021 - http://www.indexfungorum.org/names/Names.asp (Accessed on April 15, 2021)

Jatta A. 1911 - Lichenes. Flora ItalicaCryptogama. Pars. III. Rocca S. Cassiano.

Jayasiri SC, Hyde KD, Ariyawansa HA, Bhat J et al. 2015 - The Faces of Fungi database: fungal names linked with morphology, phylogeny and human impacts. Fungal Diversity 74, 3-18.

Katoh K, Rozewicki J, Yamada KD. 2019 - MAFFT online service: multiple sequence alignment, interactive sequence choice and visualization. Briefings in bioinformatics 20(4), 1160-1166. 
Lindau G. 1897 - Pyrenomycetineae. - in: A. Englek \& K. Prantl, Natürl. Pflanzenfam 1(1), 321505.

Lipnicki LI. 2015 - The role of symbiosis in the transition of some eukaryotes from aquatic to terrestrial environments. Symbiosis 65, 39-53.

Liu JK, Phookamsak R, Dai DQ, Tanaka K et al. 2014 - Roussoellaceae, a new pleosporalean family to accommodate the genera Neoroussoella gen. nov., Roussoella and Roussoellopsis. Phytotaxa 181, 1-33.

Lücking R, Hodkinson BP, Leavitt SD. 2017 - The 2016 classification of lichenized fungi in the Ascomycota and Basidiomycota - Approaching one thousand genera. The Bryologist 119(4), 361-416.

Lücking R, Nelsen MP, Aptroot A, Benatti MN et al. 2016a - A pot-pourri of new species of Trypetheliaceae resulting from molecular phylogenetic studies. Lichenologist 48(6), 639660.

Lücking R, Nelsen MP, Aptroot A, De Klee RB et al. 2016b - A phylogenetic framework for reassessing generic concepts and species delimitation in the lichenized family Trypetheliaceae (Ascomycota: Dothideomycetes). Lichenologist 48(6), 739-762.

Lutzoni F, Pagel M, Reeb V. 2001 - Major fungal lineages are derived from lichen symbiotic ancestors. Nature 411(6840), 937-940.

Massalongo AB. 1852 - Ricerche Sull'autonomia Dei Licheni Crostosi e materiali pella loro naturale ordinazione. Frizerio, Verona

Massalongo AB. 1854 - Gineacaena lichenum noviter proposita ac descripta. Ramanzini.

Miller MA, Pfeiffer W, Schwartz T. 2010 - Creating the CIPRES Science Gateway for inference of large phylogenetic trees: Proceedings of the Gateway Computing Environments Workshop (GCE) $1-8$.

Müller J. 1883 - Lichenologische Beiträge XVIII. Flora 66(16), 243-249.

Nelsen MP, Lücking R, Aptroot A, Andrew CJ et al. 2014 - Elucidating phylogenetic relationships and genus-level classification within the fungal family Trypetheliaceae (Ascomycota: Dothideomycetes). Taxon 63, 974-992.

Nelsen MP, Lücking R, Boyce CK, Lumbsch HT et al. 2020 - The macroevolutionary dynamics of symbiotic and phenotypic diversification in lichens. Proceedings of the National Academy of Sciences 117(35), 21495-21503.

Nelsen MP, Lücking R, Grube M, Mbatchou JS et al. 2009 - Unravelling the phylogenetic relationships of lichenised fungi in Dothideomyceta. Studies in Mycology 64, 135-144.

Nelsen MP, Lücking R, Mbatchou JS, Andrew CJ et al. 2011 - New insights into relationships of lichen-forming Dothideomycetes. Fungal Diversity 51(1), 155-162.

Nylander JAA. 2004 - MrAIC. pl. Program distributed by the author. Sweden: Evolutionary Biology Centre, Uppsala University.

Pérez-Ortega S, Garrido-Benavent I, Grube M, Olmo R et al. 2016 - Hidden diversity of marine borderline lichens and a new order of fungi: Collemopsidiales (Dothideomyceta). Fungal Diversity 80(1), 285-300.

Pinnoi A, Jeewon R, Sakayaroj J, Hyde KD et al. 2007 - Berkleasmium crunisia sp. nov. and its phylogenetic affinities to the Pleosporales based on $18 \mathrm{~S}$ and $28 \mathrm{~S}$ rDNA sequence analyses. Mycologia 99(3), 378-384.

Rambaut A. 2014 - FigTree v1.4: Tree figure drawing tool. http://tree. bio.ed.ac.uk/software/figtree/ (Accessed on March 10, 2021).

Sar P, Zhang Y, Wang HK, Fournier J et al. 2009 - Towards a phylogenetic clarification of Lophiostoma/Massarina and morphologically similar genera in the Pleosporales. Fungal Diversity 225-251.

Schaerer LE. 1836 - Lichenum Helveticorum Spicilegium. Pars Prima, Sectiones I-VII. Oficina Halleriana, Bernae.

Schoch CL, Crous PW, Groenewald JZ, Boehm EWA et al. 2009 - A class-wide phylogenetic assessment of Dothideomycetes. Studies in Mycology 64, 1-15. 
Species Fungorum. 2021 - http://www.speciesfungorum.org/Names/Names.asp (Accessed on April $15,2021)$

Stamatakis A. 2014 - RAxML version 8: a tool for phylogenetic analysis and post-analysis of large phylogenies. Bioinformatics 30, 1312-1313.

Thiyagaraja V, Lücking R, Ertz D, Karunarathna SC et al. 2021 - The Evolution of life modes in Stictidaceae, with three novel taxa. Journal of Fungi 7(2), 105.

Thiyagaraja V, Lücking R, Ertz D, Wanasinghe DN et al. 2020 - Evolution of non-lichenized, saprotrophic species of Arthonia (Ascomycota, Arthoniales) and resurrection of Naevia, with notes on Mycoporum. Fungal Diversity 102(1), 205-224.

Tucker SC, Harris RC. 1980 - New and noteworthy pyrenocarpous lichens from Louisiana and Florida. Bryologist 1-20.

Vainio E. 1921 - Lichenes ab A. Yasuda in Japoniacollecti. Continuatio I. Bot Mag (Tokyo) 25, 45-79

Vilgalys R, Hester M. 1990 - Rapid genetic identification and mapping of enzymatically amplified ribosomal DNA from several Cryptococcus species. Journal of bacteriology 172, 4238-4246.

von Arx JA, Müller E. 1975 - A re-evaluation of the bitunicate Ascomycetes with keys to families and genera. Studies in Mycology 9, 1-159

Watson W. 1929 - The classification of lichens. New Phytologist 28(2), 85-116.

Wijayawardene NN, Hyde KD, Al-Ani LKT, Tedersoo L et al. 2020 - Outline of Fungi and fungus-like taxa. Mycosphere 11(1), 1060-1456.

Wijayawardene NN, Hyde KD, Lumbsch HT, Liu JK et al. 2018 - Outline of Ascomycota: 2017. Fungal Diversity 88, 167-263.

Wijayawardene NN, Hyde KD, Rajeshkumar KC, Hawksworth DL et al. 2017 - Notes for genera: Ascomycota. Fungal Diversity 86, 1-594.

Yu Y, Blair C, He X. 2020 - RASP 4: ancestral state reconstruction tool for multiple genes and characters. Molecular Biology and Evolution 37(2), 604-606.

Yu Y, Harris AJ, Blair C, He X. 2015 - RASP (Reconstruct Ancestral State in Phylogenies): a tool for historical biogeography. Molecular phylogenetics and evolution 87, 46-49.

Zahlbruckner A. 1921 - Catalogus Lichenum Universalis Volume I. Leipzig: Borntraeger.

Zhang Y, Jeewon R, Fournier J, Hyde KD. 2008 - Multi-gene phylogeny and morphotaxonomy of Amniculicola lignicola: a novel freshwater fungus from France and its relationships to the Pleosporales. Mycological Research 112(10), 1186-1194.

Zoller S, Scheidegger C, Sperisen C. 1999 - PCR primers for the amplification of mitochondrial small subunit ribosomal DNA of lichen-forming ascomycetes. Lichenologist 31(5), 511-516. 